\title{
Development of real-time laser-scanning system to detect tree canopy characteristics for variable-rate pesticide application
}

\author{
Cai Jichen ${ }^{1,2,3,4}$, Wang $\mathrm{Xiu}^{2,3,4}$, Song Jian ${ }^{2,3,4}$, Wang Songlin ${ }^{2,3,4}$, \\ Yang Shuo 2,3,4 Zhao Chunjiang ${ }^{1,2,3,4 *}$ \\ (1. College of Information and Electrical Engineering, China Agricultural University, Beijing 100083, China; \\ 2. National Research Center of Intelligent Equipment for Agriculture, Beijing 100097, China; \\ 3. Key Laboratory of Agri-informatics, Ministry of Agriculture, Beijing 100097, China; \\ 4. Beijing Key Laboratory of Intelligent Equipment Technology for Agriculture, Beijing 100097, China)
}

\begin{abstract}
Improving pesticide application efficiency is increasingly important in orchard spraying. In this study, a laser-scanning system was designed to acquire gridding volumes of a tree to quantify the geometry characteristics of the tree canopy in real-time. A laser-scanning sensor mounted on a linear guide was utilized to measure the structure of a target tree canopy. A computer was used to receive measurement data from the laser scanner and obtain the movement distance of the laser-scanning sensor from a controller. An algorithm written with VC\# program was designed to calculate gridding volumes of trees by recognizing valid measurement data from the laser scanner. Laboratory evaluations were conducted on three kinds of regular objects, and the maximum relative errors of section volumes of the cuboid, triangular prism and cylinder objects were $3.3 \%, 7.9 \%$ and $9.4 \%$, respectively, which illustrated that the algorithm could calculate the section volumes in different parts of the objects with high accuracy. A conifer tree and an apple tree were chosen to verify detecting accuracy of the laser-scanning system at variable speeds and grid sizes. The variation coefficients of total volumes for each kind of the tree were 0.078 and 0.041, respectively, which indicated that the laser-scanning system could be applied to provide the gridding volumes of different canopy densities in real-time with good reliability for guiding a variable-rate sprayer.
\end{abstract}

Keywords: laser-scanning system, sensor, tree canopy, variable-rate spray, pesticide application efficiency, gridding volumes, calculation algorithm

DOI: $10.25165 /$ j.ijabe. 20171006.3140

Citation: Cai J C, Wang X, Song J, Wang S L, Yang S, Zhao C J. Development of real-time laser-scanning system to detect tree canopy characteristics for variable-rate pesticide application. Int J Agric \& Biol Eng, 2017; 10(6): 155-163.

\section{Introduction}

Improving pesticide application efficiency is more

\footnotetext{
Received date: 2016-12-18 Accepted date: 2017-09-10

Biographies: Cai Jichen, $\mathrm{PhD}$ candidate, research interests: intelligent equipment for agriculture, Email: caijichen@foxmail. com; Wang Xiu, PhD, Professor, research interests: intelligent equipment for fertilizer and pesticide on agriculture, Email: wangx@nercita.org.cn; Song Jian, Engineer, research interests: agricultural information technology, Email: songj@nercita.org.cn; Wang Songlin, $\mathrm{PhD}$ candidate, research interests: intelligent equipment for agriculture, Email: wangsl@ nercita.org.cn; Yang Shuo, Master candidate, research interests: intelligent equipment for agriculture, Email: yangshuo@foxmail.com.

*Corresponding Author: Zhao Chunjiang, PhD, Professor, CAE Academician, majored in agricultural information technology. Beijing Research Center of Intelligent Equipment for Agriculture, Beijing 100097, China. Email: zhaocj@ nercita.org.cn.
}

important with the development of precision agriculture research in an orchard. A sprayer with variable-rate spray functions ${ }^{[1-5]}$ makes it possible to reduce pesticide applications, decrease chemical deposits on the ground, and minimize environmental pollution.

In recent years, tree detection sensors for variable-rate sprayers, mainly including infrared sensors, ultrasonic sensors and laser scanners, have been used in measuring tree canopy characteristics such as canopy porosity and foliage density ${ }^{[6]}$. Zhai et al. ${ }^{[7-9]}$ verified the little influence of velocity on orchard tree volume detection using ultrasonic sensor and proposed a method for estimating canopy density with a detecting system based on an ultrasonic sensor. Kang et al. ${ }^{[10-12]}$ developed a sucker detection method based on information fusion 
using laser scanner and machine vision to identify and locate grapevine sucker with high recognition rate and relative position accuracies, proposed a targeted spray application for grapevine sucker control in grape vineyards.

Rosell et al. ${ }^{[13-15]}$ used a laser scanner to estimate 3D structure and canopy surface of trees, and realized tree reconstruction based on point cloud data with a short processing time using the proposed algorithm. $\mathrm{Yu}$ et al. $^{[16,17]}$ built a platform for measuring the fruit tree canopy volume by using a laser-scanning sensor combined with AHRS and DGPS sensors. They all obtained tree canopy volumes with small relative measuring errors, comparing with manual measurements. However, all of the measurement data needed to be analyzed after being collected, which would make the spraying work complicated.

Meanwhile, three-dimensional (3D) laser-scanning sensors were also applied to estimate tree canopy volume in recent years ${ }^{[18,19]}$. It could be used to offer high-precision measurement data and build 3D models of tree canopies by the laser point cloud in the geodetic coordinate system. However, it is not only time-consuming for the 3D laser-scanning sensor during the data post processing, but also too expensive to be applied for spraying work in an orchard.

Chen et al. ${ }^{[20,21]}$ developed a variable-rate air-assisted sprayer prototype with a laser-scanning sensor to control the flow rate of individual nozzles depending on the foliage density in real-time. The intelligent sprayer could detect not only the foliage density, but also the canopy height, width, volume and occurrence of one side. Osterman et al. ${ }^{[22]}$ proposed a canopy boundarysmoothing algorithm for positioning of spraying arms which were mounted on an orchard sprayer based on a laser-scanning system. However, the scanning system needed to work at a constant speed since the sprayer did not have a speed sensor to measure the travel speed in real-time. Llorens et al., Gamarra-Diezma et al. and Miranda-Fuentes et al. ${ }^{[23-25]}$ developed a variable-rate sprayer prototype with ultrasonic and laser sensors, and compared the accuracy of both detecting methods, concluding that improving the method of data processing was still needed while a laser scanner could provide more accuracy and detailed information about the canopy.

In order to enable the sprayer to provide uniform spray deposition across an entire tree canopy, its canopy structure was divided into sections, and each section received a certain amount of pesticide based on its volume or foliage density. The objective of this research was to develop a system with an optimized calculating algorithm to estimate the volume of each section in the tree canopy grid at variable moving speeds.

\section{Materials and methods}

\subsection{Design of detecting system}

The used detecting system whose structure diagram is shown in Figure 1 mainly included a laser-scanning sensor, a linear guide, an incremental encoder, a servo motor control system (controller) and a computer. The high-speed laser-scanning sensor (LMS511, SICK, Inc., Germany) was mounted on a linear slider of the $2 \mathrm{~m}$ long linear guide to scan target trees vertically to characterize canopy structures. The linear slider was driven by a servo motor with a speed range of $0-5.4 \mathrm{~km} / \mathrm{h}$ using the controller to simulate the movement of a tractor. The height of the laser-scanning sensor could be adjusted on the linear guide. The incremental encoder was used to measure the distance that the laser scanner travelled, and transmit the pulse signals to the controller. The computer was used to obtain the movement distance data of the laser scanner from the controller, receive measurement data from the laser scanner, calculate the volumes of all sections, and send spray data to the controller in real-time.

A gridding volumes calculating software was developed using C\# language which could run on a computer. The scanning parameters on the interface included the distance between the laser-scanning sensor and tree row plane, the scanner height, the height and the width of each canopy section covered by each nozzle, and the distance between laser-scanning sensor and nozzles. After the parameters set up, the computer communicated with the laser-scanning sensor at a certain scanning mode, received a stream of measurement data continuously, manipulated the range data stream and calculated section volumes, displayed the gridding volumes data on the interface and saved them in an excel file at the same time. 


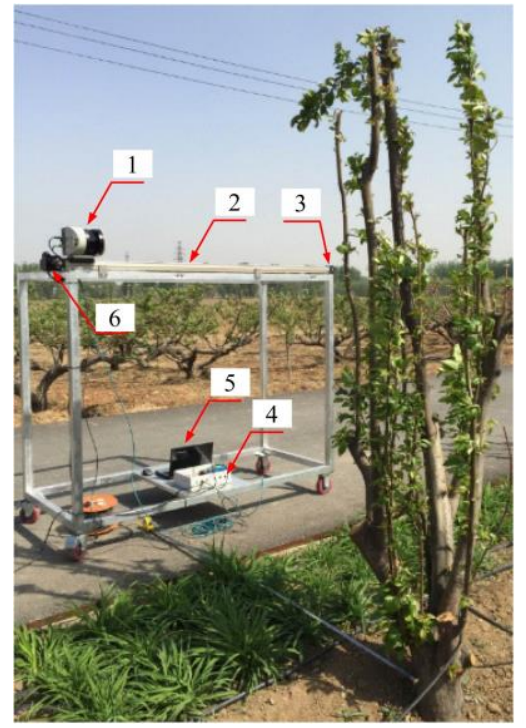

a. Picture of the laser-scanning system

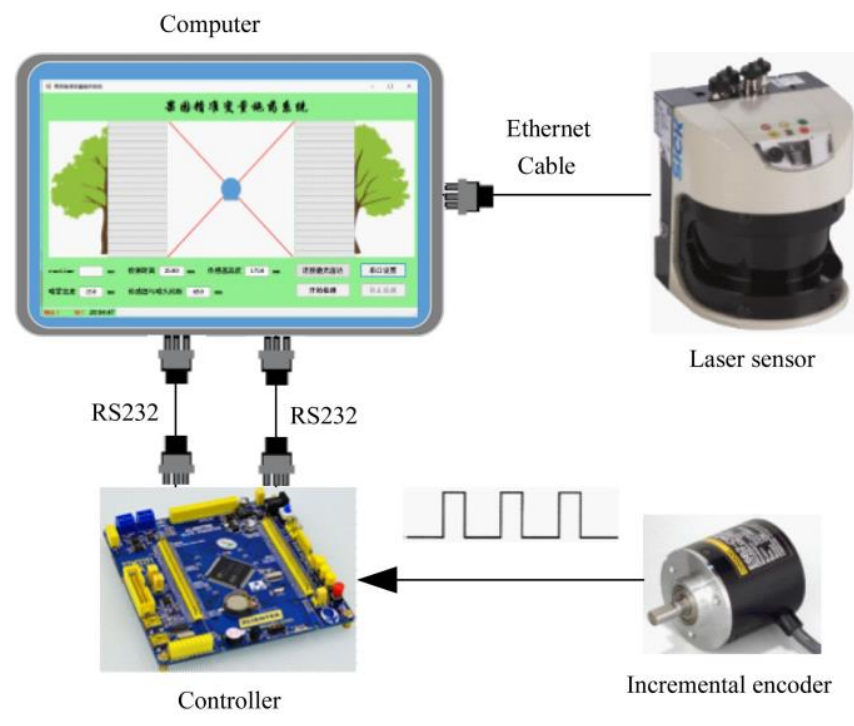

b. Schematic diagram of the detection system

1. Laser-scanning sensor 2. Linear Slider 3. Incremental encoder 4. Controller 5. Computer 6. Servo Motor

Figure 1 Structure diagram of laser-scanning system

In order to transmit measurement data at maximum speed, the laser scanner and computer were connected with an Ethernet cable, because it was the only way to output all measured data of each scan in real-time using an Ethernet interface. The data transmission rate was $100 \mathrm{Mbit} / \mathrm{s}$. The laser-scanning sensor could continuously transmit and receive 361 signals in a $180^{\circ}$ radial range with $0.5^{\circ}$ angular resolution within a $20 \mathrm{~ms}$ measurement cycle. In this study, the sensor was tuned to receive 241 signals from $30^{\circ}$ to $150^{\circ}$ with $0.5^{\circ}$ angular resolution to improve the system operation speed. In the program, a timer was inserted at the beginning of receiving the movement distance data from the controller. And the amount of time consumed by each processing cycle would be calculated after showing the gridding volumes data on the interface. The each processing time was no more than $30 \mathrm{~ms}$, which would meet the demand of dividing a tree canopy into small sections when a sprayer traveled at normal working speed.

\subsection{Measurement data acquisition and preprocessing}

When the laser-scanning sensor traveled on the linear guide, the incremental encoder transmitted pulse signals to the controller. And then, the controller accumulated the pulse signals and sent the movement distance, which represented the width of the canopy grid $(w)$, to the computer. After that, the computer asked for a measurement data from the laser-scanning sensor. The tree canopy profile facing to the laser-scanning sensor was detected and all of the measurement data were assembled into a measurement data string which was then sent to the computer.

In this procedure, the header of each measurement data string was firstly identified, and then the expected amount of the measurement data was parsed. If an error data string was received, the program would discard the string and begin to receive next one until every measurement datum was read in accordance with the order of the angle increment. The canopy depth $\left(d_{i}\right)$ between the point detected on the canopy surface and the tree row center, and the height of each point $\left(h_{i}\right)$ was calculated according to the measured data and angles. Subsequently, unwanted measurement data from the ground, sky, or objects beyond the targeted tree row plane would be filtered by comparing the canopy depth $\left(d_{i}\right)$ with preset threshold. Finally, a new string, including the canopy depth $\left(d_{i}\right)$ and the height of each point $\left(h_{i}\right)$, was created for later calculation of the canopy volume.

\subsection{Algorithm design}

In this study, 'section volume' as labeled in the red box $\left(V_{k}\right.$ or $\left.V_{n}\right)$ in Figure 2, represented the enclosed space between canopy profile and the tree row plane. The detected tree canopy was equally divided into multiple sections in the vertical direction with a certain height; therefore, each section covered a varied number $\left(N_{k}\right)$ of 
data points. The program would organize the measurement data for each section to calculate gridding volumes.

Tree canopy includes foliage materials (leaves and branches) and empty spaces ${ }^{[20]}$. It is very common that the laser beam goes through the tree (point $\mathrm{C}$, which would be filtered in preprocessing) from empty spaces, or goes into the canopy (point D) because of the lack of leaves. As a result, two adjacent data points (points A, $\mathrm{B}$, points $\mathrm{D}, \mathrm{E}$, which were read in the measurement data string one by one) would exist two situations. One situation is that $h_{i+1}$ (the height of point $\mathrm{B}$ ) is higher than $h_{i}$ (the height of point A). The other one is that the $h_{D}$ (the height of point D) could be higher than or equal to $h_{E}$ (the height of point E). Because the laser beam goes into the canopy, the second situation might lead to the measurement data read earlier located higher than the data read later. As a result, all of the measurement data in one string needed to be rearranged in order of height. In order to calculate the section volumes, all the volumes between two adjacent data points in one section needed to be accumulated. The difference of the adjacent data points' heights was used as the height of the volumes between two adjacent data points.
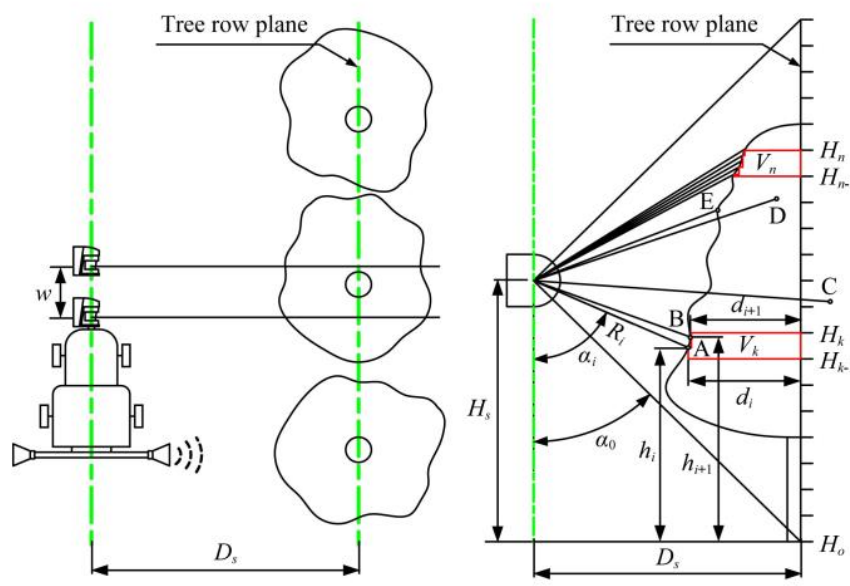

Figure 2 Top and front view representation of a scanning procedure

The formula for calculating the section volume of the canopy $\left(V_{k}\right)$ is shown as follows:

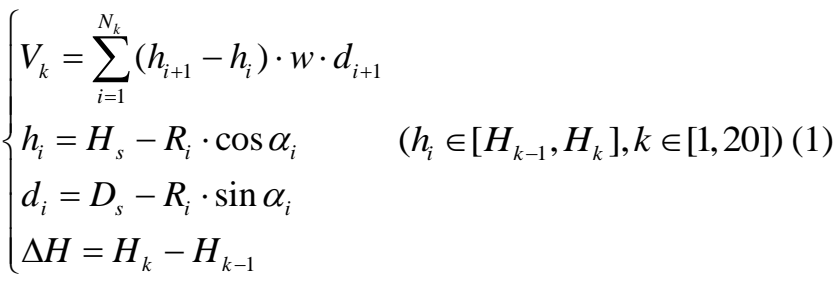

where, $i$ is the index of the data point after rearrangement in order of height; $h_{i}$ is the height of the individual data point $i, \mathrm{~mm} ; w$ is the width of a section of the canopy grid, $\mathrm{mm} ; d_{i}$ is the depth of the data point $i, \mathrm{~mm} ; H_{k}$ is the height of the section $k, \mathrm{~mm} ; N_{k}$ is the number of the data points in section $k ; H_{s}$ is the height of the laser-scanning sensor, $\mathrm{mm} ; D_{s}$ is the distance between the laser-scanning sensor and the tree row plane, $\mathrm{mm} ; R_{i}$ is the distance from the data point $i$ to the sensor, mm; $\alpha_{i}$ is the vertical angle of the laser beam $i,\left(^{\circ}\right) ; \Delta H$ is the height of a section of the canopy grid, $\mathrm{mm}$.

Finally, the program would show volumes of the sections of each scan on the screen in the form of histogram, and save them to an excel file. The program flowchart for calculating section volumes is shown in Figure 3.

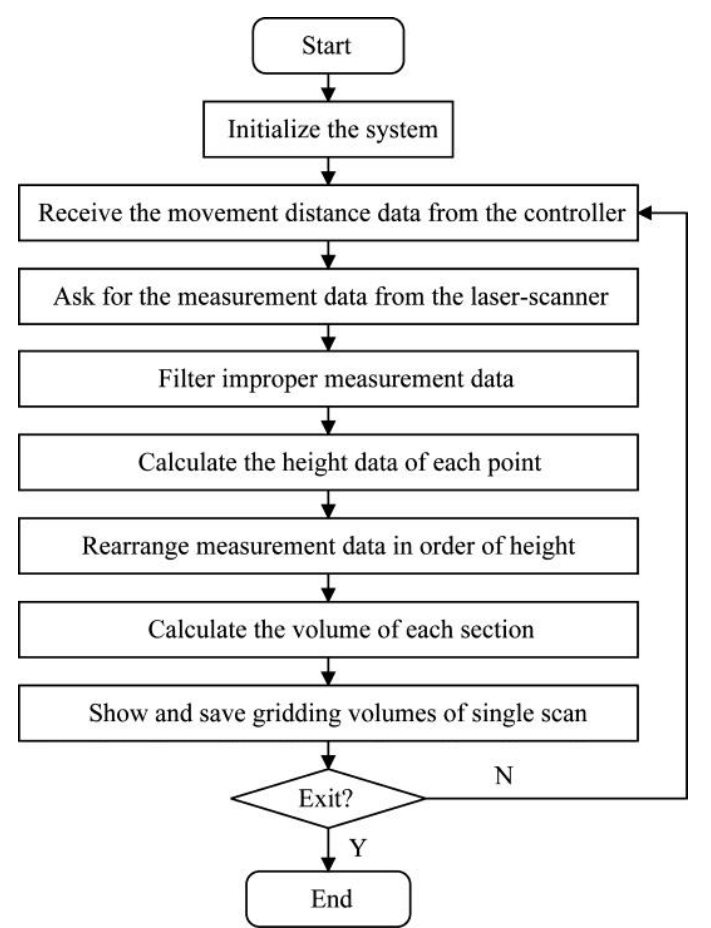

Figure 3 Program flowchart for calculating section volumes

\subsection{Experimental procedure}

In order to verify the accuracy of volume calculating of the algorithm, a cuboid (Length=560 mm; Height= $950 \mathrm{~mm}$; Thickness $=360 \mathrm{~mm}$ ), a cylinder (Diameter $=$ $580 \mathrm{~mm}$; Height=880 $\mathrm{mm}$ ), and a triangular prism (Length=710 mm; Height=1165 $\mathrm{mm}$ ) were used for testing. The test was conducted 6 times at $1.5 \mathrm{~km} / \mathrm{h}$, $3.0 \mathrm{~km} / \mathrm{h}$ and $4.5 \mathrm{~km} / \mathrm{h}$. The height $(\Delta H)$ and the width $(w)$ of each section of the canopy grid were set to be $150 \mathrm{~mm}$ and $50 \mathrm{~mm}$, respectively. The scan distance 
$\left(D_{s}\right)$ was set to be $1500 \mathrm{~mm}$; the height of the laser-scanning sensor $\left(H_{s}\right)$ was set to be $1500 \mathrm{~mm}$. During the test, the laser-scanning sensor would move forward and back on the linear guide at different speeds. The movement in one direction was one test. After each test was finished, the gird volumes saved in the excel file would be analyzed and compared with the real volumes of objects.

Two kinds of trees, a conifer tree and an apple tree, were chosen to test the total volume uniformity with the laser-scanning sensor scanning 6 times at different speeds of $1.5 \mathrm{~km} / \mathrm{h}, 3.0 \mathrm{~km} / \mathrm{h}$ and $4.5 \mathrm{~km} / \mathrm{h}$. The average wind velocity was $2.8 \mathrm{~m} / \mathrm{s}$. The conifer canopy was relatively dense. The height and maximum width of the conifer canopy were about $1300 \mathrm{~mm}$ and $500 \mathrm{~mm}$. The scan height $\left(H_{s}\right)$ and the scan distance $\left(D_{s}\right)$ were both set to be $1500 \mathrm{~mm}$. The width $(w)$ of canopy grid was set to be $50 \mathrm{~mm}, 75 \mathrm{~mm}$ and $100 \mathrm{~mm}$, respectively in scanning precision tests. The apple canopy was relatively sparse. The height and maximum width of the apple canopy were about $2900 \mathrm{~mm}$ and $1400 \mathrm{~mm}$. The scan height $\left(H_{s}\right)$ and the scan distance $\left(D_{s}\right)$ were $1700 \mathrm{~mm}$ and $1950 \mathrm{~mm}$. The width $(w)$ values of canopy grid were set to be $50 \mathrm{~mm}, 100 \mathrm{~mm}$ and $150 \mathrm{~mm}$, respectively.

\section{Results and discussion}

\subsection{Results of detecting regular objects}

The results of the gridding volumes of three regular objects which were analyzed using Matlab are presented in Figure 4. The reason why the volumes of the top sections were lower than other sections was that the top sections were not full. The results presented in Figures 4 and 5 showed that the detection gridding volumes were sharply in accordance with the shape of the objects. Each section volume ${ }^{[20]}$ was very close to its real volume, and the maximum relative errors are $3.3 \%, 7.9 \%$ and 9.4\% respectively as shown in Table 1 . However, the maximum relative errors of the objects increased gradually. That's because the shape of each divided section is not a standard cuboid due to the profile of the triangular prism while they were calculated as a cuboid. The similar thing happened when the cylinder was detected, and the volumes of some sections are larger than the triangular prism's because of the profile of the cylinder. It could also be demonstrated by the results shown in Figure 5, some parts (detection position 9 to 14 of the triangular prism; detection position 1 to 5 of the cylinder) of the section volumes are slightly larger than the actual volumes, and the others are smaller.

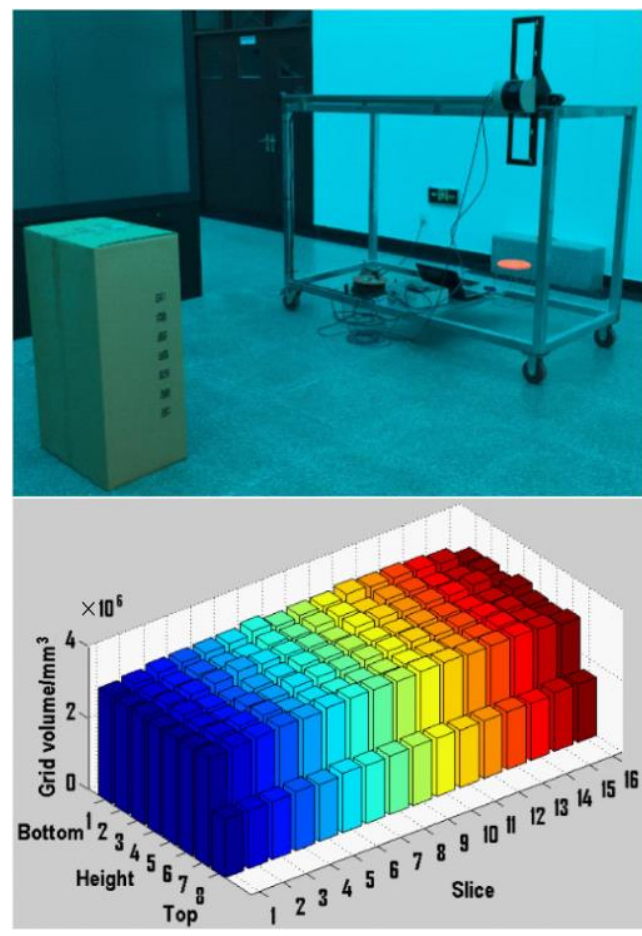

a

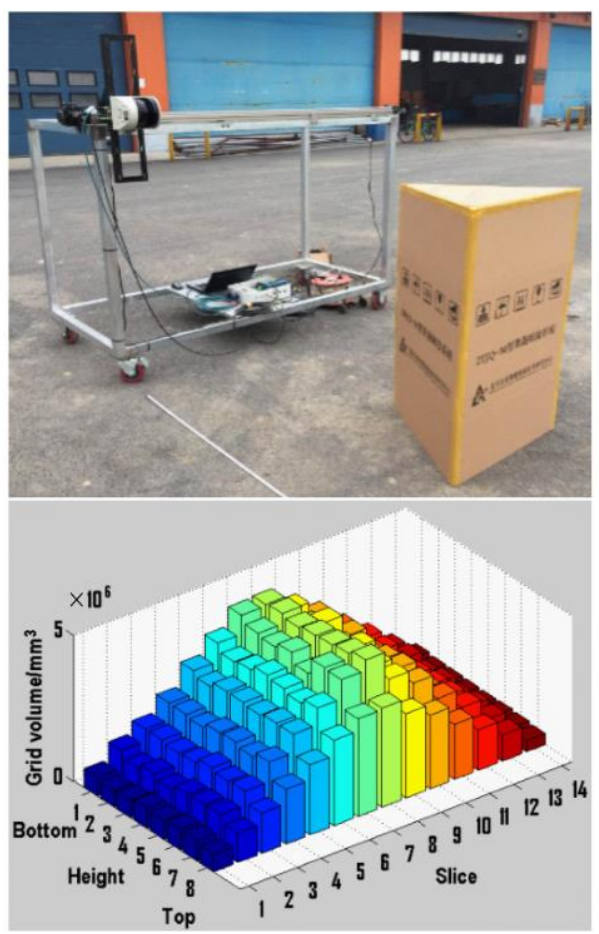

$\mathrm{b}$

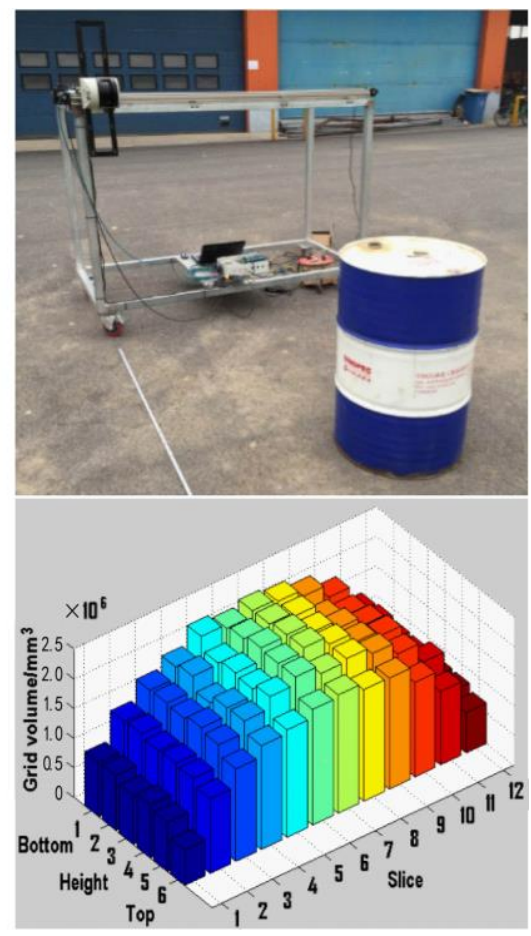

c

Figure 4 Gridding volumes of regular objects 


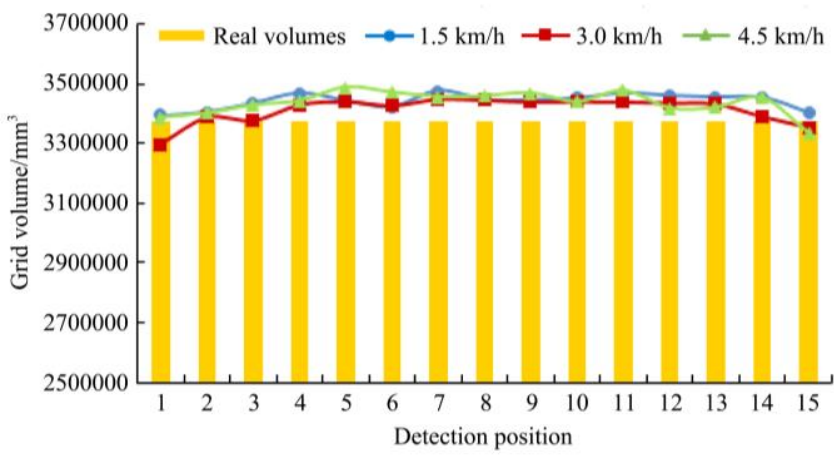

a. Cylinder object

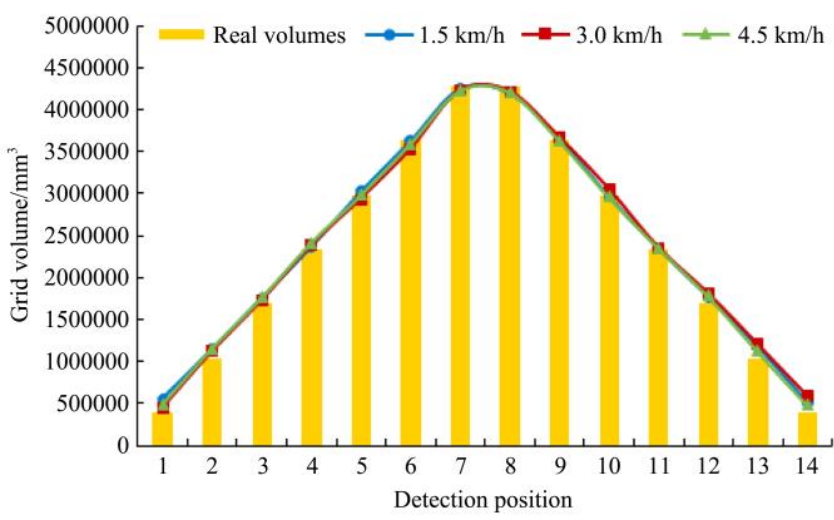

b. Triangular prism object

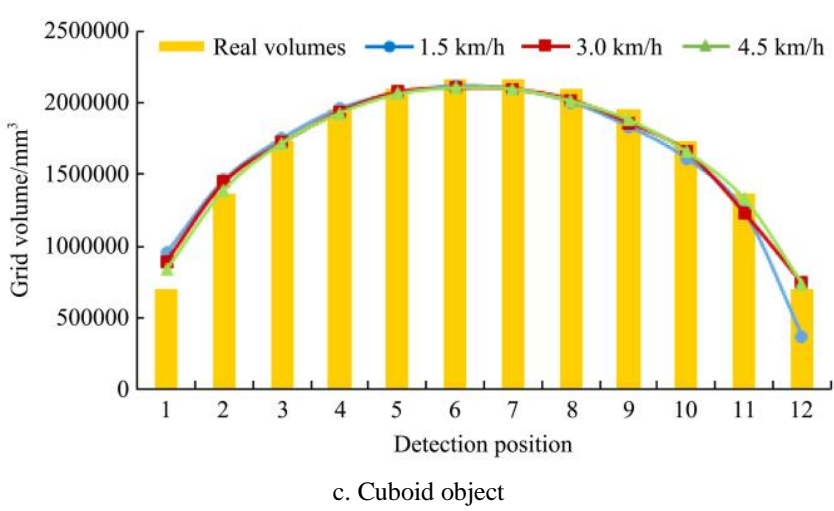

Figure 5 Comparison of the gridding volumes of regular objects at different speeds

Table 1 Maximum relative errors of gridding volumes of objects at different speeds

\begin{tabular}{|c|c|c|c|c|c|c|c|c|c|}
\hline \multirow{2}{*}{$\frac{\text { Object }}{\text { Velocity } / \mathrm{km} \cdot \mathrm{h}^{-1}}$} & \multicolumn{3}{|c|}{ Cuboid } & \multicolumn{3}{|c|}{ Triangular prism } & \multicolumn{3}{|c|}{ Cylinder } \\
\hline & 1.5 & 3.0 & 4.5 & 1.5 & 3.0 & 4.5 & 1.5 & 3.0 & 4.5 \\
\hline $\begin{array}{l}\text { Max. relative } \\
\text { error } / \%\end{array}$ & 2.9 & 2.2 & 3.3 & 5.1 & 7.3 & 5.3 & 8.9 & 9.4 & 4.1 \\
\hline
\end{tabular}

As shown in Figure 5, the relative errors of section volumes of the cuboid objects in different parts had no big difference, but the relative errors of section volumes on both sides of the edge of cylinder and triangular prism objects were little bigger than them in the center. The deviation of the section volume was bigger at the edge of the objects mainly because the deviation of the scan position at each test. In addition, the systematic measurement error of laser-scanning sensor which was $\pm 30 \mathrm{~mm}$ typically, and the measurement of the scan height $\left(H_{s}\right)$ and the scan distance $\left(D_{s}\right)$ with steel tape made the deviation either. The results demonstrated that the algorithm for the control system was able to calculate the volumes of the sections with little variation.

The total volumes of the regular objects detected using the laser-scanning sensor at different travel speeds are shown in Figure 6. The total volumes detected under different detecting speeds were very close, with maximum relative error less than $5 \%$. It illustrated that the algorithm for the scanning system was able to obtain the total volume of the regular object and the volume of each section precisely at different speeds.

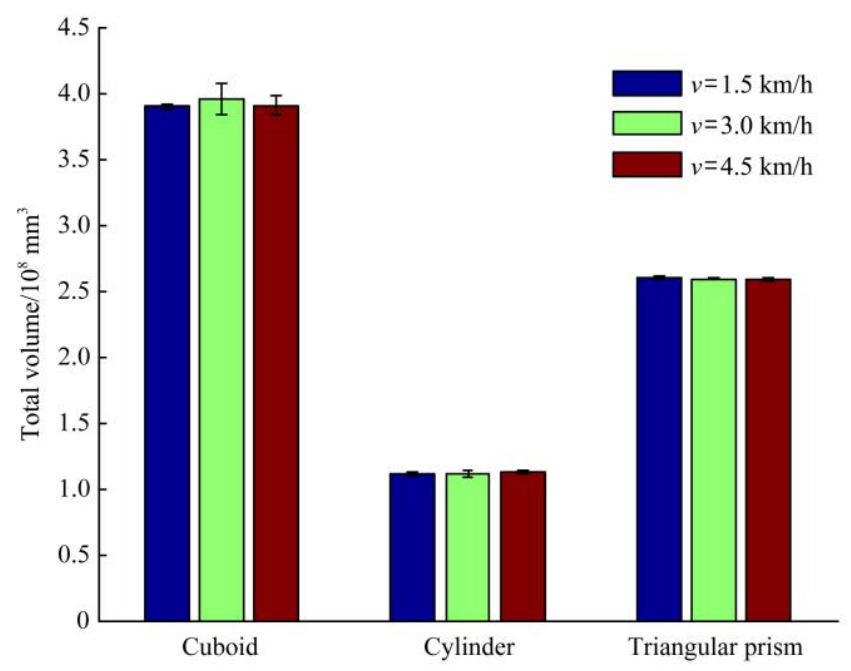

Figure 6 Total volumes of the regular objects detected at different speeds

\subsection{Results of detecting individual trees}

The gridding volumes for two kinds of trees detected using the laser-scanning sensor at different speeds are shown in Figure 7. The section volumes of the trees were analyzed using Matlab. The trees' canopies detected under different grid sizes were similar, which demonstrated that the algorithm could characterize trees' canopy structures stably. And this is one of the important design criteria of the detecting system for variable-rate spraying. Some section volumes of two kinds of trees had variation probably because the laser-scanning sensor started to scan the apple tree at different locations and the branch was slightly swaying in the wind during the test.

The average total volumes of each individual tree detected using the laser-scanning sensor at different grid 
sizes and speeds are shown in Figure 8. There was also no big difference in the total volumes of the trees under different detecting circumstances, which also showed that the velocity had little influence on orchard tree volume detection $^{[21]}$. However, the maximum variances of the total volumes detected at different grid sizes increased gradually. It illustrated that the detecting accuracy would decrease when the grid sizes were getting bigger. The coefficients of variation of the total volumes of the two kinds of trees were 0.078 and 0.041 respectively, which indicated that the algorithm could calculate tree canopy volumes with high repetitive detection accuracy.
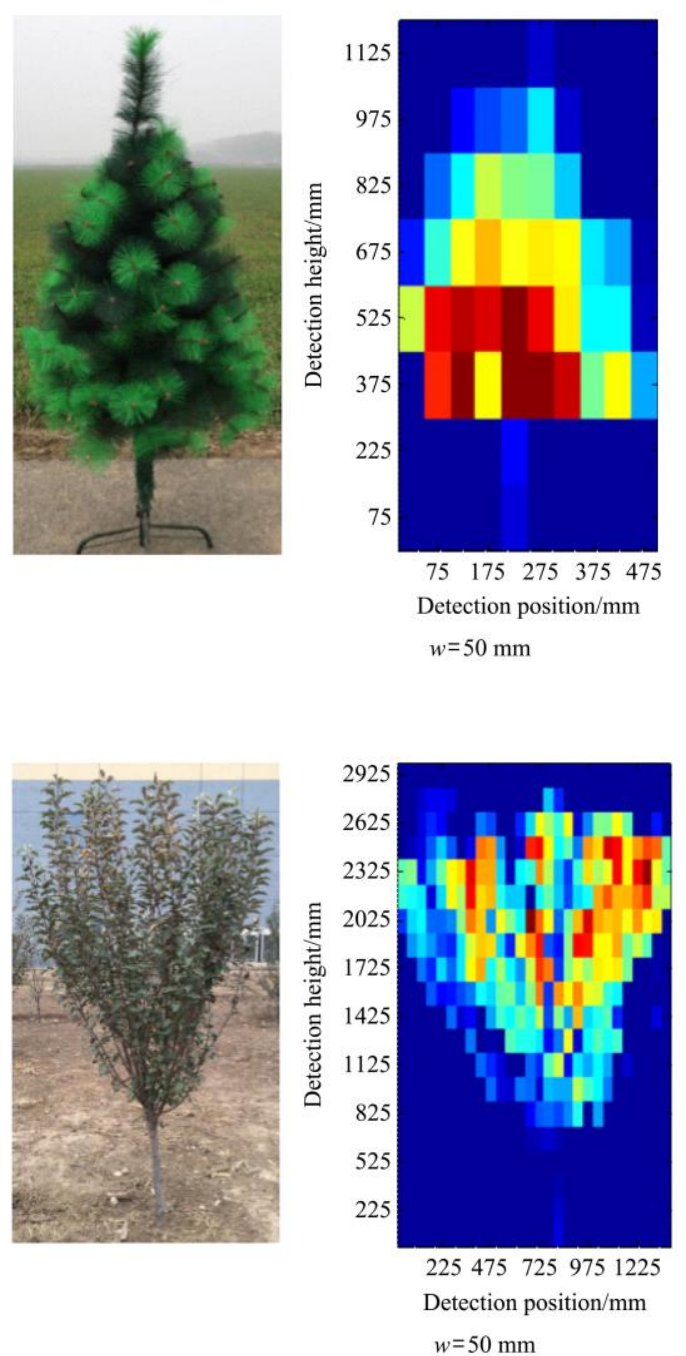

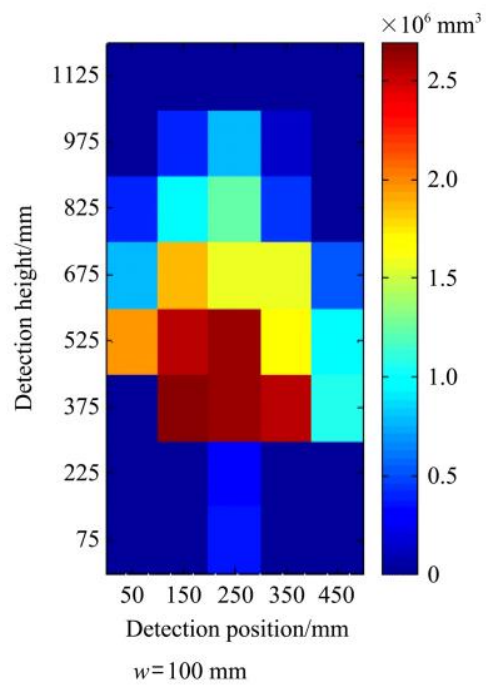

a. Conifer tree
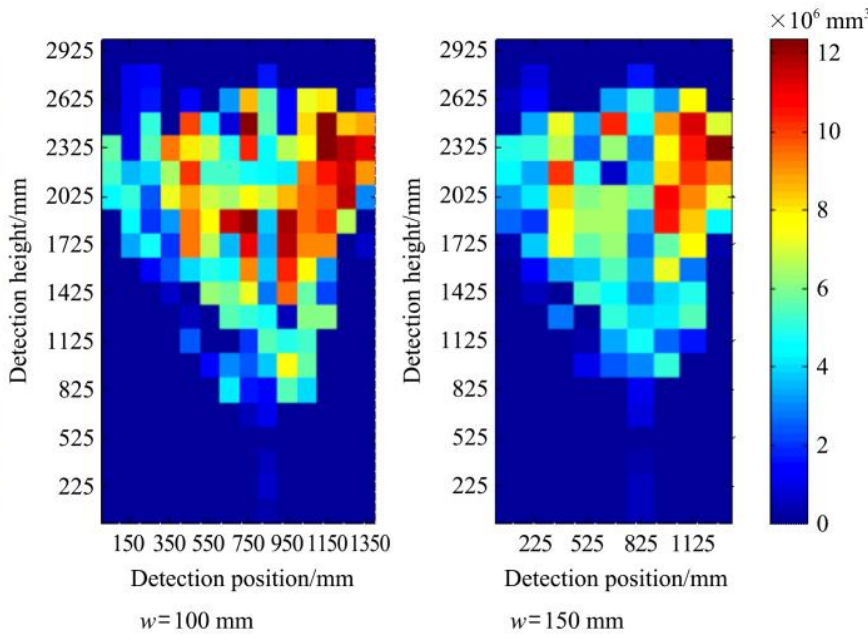

b. Apple tree

Figure 7 Gridding volumes of trees under different grid size
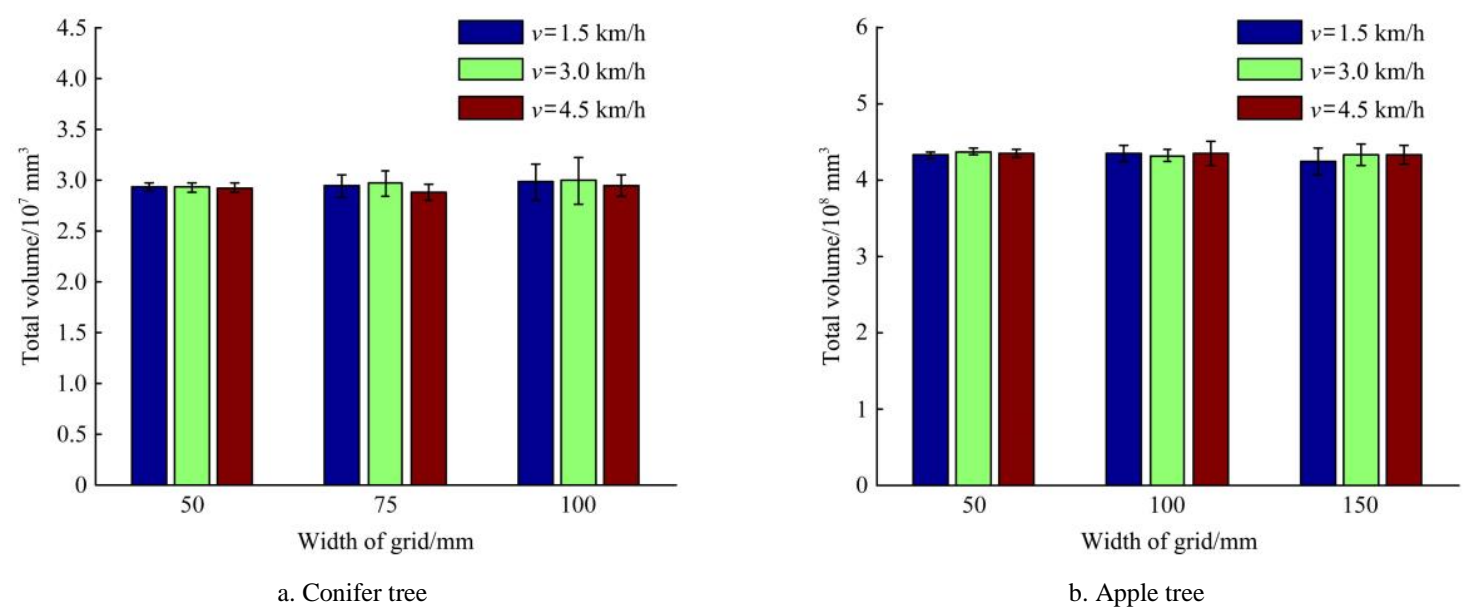

Figure 8 Average total volumes of trees detected at different grid sizes and speeds 


\section{Conclusions}

A fast algorithm was developed to calculate tree canopy gridding volumes, which could be applied at various speeds. The tests with the regular objects were conducted to validate the accuracy of the algorithm for calculating gridding volumes. Results showed that the maximum relative errors of section volumes of the cuboid, triangular prism and cylinder objects were 3.3\%, 7.9\% and $9.4 \%$, respectively. The errors of the total volumes were less than 5\% at different travel speeds. It indicated that the velocity had little influence on calculating the section volumes in different parts of the objects.

The experiment for two kinds of trees showed that the detecting system could characterize tree canopy structures of different densities with good reliability. The variation coefficients of the total volumes of the two kinds of tree were 0.078 and 0.041 , respectively. It indicated that the laser-scanning system could provide a good estimation of the canopy volume in real-time for guiding a variable-rate sprayer.

\section{Acknowledgements}

This study was financially supported by the National Key Research and Development Program (No.2016YFD0200700).

\section{[References]}

[1] Sun H, Li M Z, Zhang Q. Detection system of smart sprayers: Status, challenges, and perspectives. Int J Agric \& Biol Eng, 2012; 5(3): 10-23.

[2] Rosell J R, Sanz R. A review of methods and applications of the geometric characterization of tree crops in agricultural activities. Computers \& Electronics in Agriculture, 2012; 81(4): 124-141.

[3] Gil E, Arnó J, Llorens J, Sanz R, Llop J, Rosell J R, et al. Advanced technologies for the improvement of spray application techniques in Spanish viticulture: An overview. Sensors, 2014; 14(1): 691-708.

[4] Song Y, Sun H, Li M, Zhang Q. Technology application of smart spray in agriculture: A review. Intelligent Automation and Soft Computing, 2015; 21(3): 319-333.

[5] Berk P, Hocevar M, Stajnko D, Belsak A. Development of alternative plant protection product application techniques in orchards, based on measurement sensing systems: A review.
Computers and Electronics in Agriculture, 2016; 124: 273-288.

[6] Zhai C Y, Zhao C J, Wang X, Liu Y Z, Ge J S, Ma Y B. Design and experiment of young tree target detector. Transactions of the CSAE, 2012; 28(2): 18-22. (in Chinese)

[7] Zhai C Y, Wang X, Zhao C J, Zou W, Liu D Y, Mao Y J. Orchard tree structure digital test system and its application. Mathematical \& Computer Modelling, 2011; 54(3-4): 1145-1150.

[8] Zhai C Y, Wang X, Guo J J, Xu S, Ma W. Influence of velocity on ultrasonic probing of orchard tree profile. Sensor Letters, 2013; 11(6): 1062-1068.

[9] Li H Z, Zhai C Y, Weckler P, Wang N, Yang S, Zhang B. A canopy density model for planar orchard target detection based on ultrasonic sensors. Sensors, 2016; 17(1): 31.

[10] Kang F, Pierce F J, Walsh D B, Zhang Q, Wang S. An automated trailer sprayer system for targeted control of cutworm in vineyards. Transactions of the ASABE, 2011; 54(4): 1511-1519.

[11] Kang F, Wang H, Pierce F J, Zhang Q, Wang S. Sucker Detection of grapevines for targeted spray using optical sensors. Transactions of the ASABE, 2012; 55(5): 2007-2014.

[12] Wang Y X, Xu S S, Li W B, Kang F, Zheng Y J. Identification and location of grapevine sucker based on information fusion of 2D laser scanner and machine vision. Int J Agric \& Biol Eng, 2017; 10(2): 84-93.

[13] Palleja T, Tresanchez M, Teixido M, Sanz R, Rosell J R, Palacin J. Sensitivity of tree volume measurement to trajectory errors from a terrestrial LIDAR scanner. Agricultural \& Forest Meteorology, 2010; 150(11): 1420-1427.

[14] Sanz R, Rosell J R, Llorens J, Gil E, Planas S. Relationship between tree row LIDAR-volume and leaf area density for fruit orchards and vineyards obtained with a LIDAR 3D Dynamic Measurement System. Agricultural \& Forest Meteorology, 2013; s171-172(3): 153-162.

[15] Méndez V, Rosell J R, Sanz R, Escolà A, Catalán H. Deciduous tree reconstruction algorithm based on cylinder fitting from mobile terrestrial laser scanned point clouds. Biosystems Engineering, 2014; 124(4): 78-88.

[16] Zhang L, Zhao Z X, Yu L, Zhang Z G, Huang J. Positioning algorithm for ultrasonic scanning of fruit tree canopy and its tests. Transactions of the CSAE, 2010; 26(9): 192-197. (in Chinese)

[17] Long Y, Jian H, Zhao Z X, Zhang L, Sun D Z. Laser measurement and experiment of hilly fruit tree canopy volume. Transactions of the CSAM, 2013; 44(8): 224-228. (in Chinese)

[18] Fernández-Sarría A, Martínez L, Velázquez-Martí B, Sajdak M, Estornell J, Recio J A. Different methodologies for calculating crown volumes of Platanus hispanica trees using 
terrestrial laser scanner and a comparison with classical dendrometric measurements. Computers \& Electronics in Agriculture, 2013; 90: 176-185.

[19] Xu W H, Feng Z K, Su Z F, Xu H, Jiao Y Q, Deng O. An automatic extraction algorithm for individual tree crown projection area and volume based on 3D point cloud data, Spectroscopy and Spectral Analysis, 2014; (2): 465-471.

[20] Chen Y. Development of an intelligent sprayer to optimize pesticide applications in nurseries and orchards. $\mathrm{PhD}$ thesis, Ohio State University, USA 2010.

[21] Chen Y, Zhu H, Ozkan H E. Development of a variable-rate sprayer with laser scanning sensor to synchronize spray outputs to tree structures. Transactions of the ASABE, 2012; 55(3): 773-781.

[22] Osterman A, Godeša $T$, Hočevar M, Sirok B, Stopar M.
Real-time positioning algorithm for variable-geometry air-assisted orchard sprayer. Computers \& Electronics in Agriculture, 2013; 98(7): 175-182.

[23] Llorens J, Gil E, Llop J, Escolà A. Ultrasonic and LIDAR sensors for electronic canopy characterization in vineyards: Advances to improve pesticide application methods. Sensors, 2011; 11(2): 2177.

[24] Gamarra-Diezma J L, Miranda-Fuentes A, Llorens J, Cuenca A, Blanco-Roldán G L, Rodríguez-Lizana A. Testing accuracy of long-range ultrasonic sensors for olive tree canopy measurements. Sensors, 2015; 15(2): 2902-2919.4

[25] Miranda-Fuentes A, Llorens J, Gamarra-Diezma J L, Gilribes J A, Gil E. Towards an optimized method of olive tree crown volume measurement. Sensors, 2015; 15(2): 3671-3687. 